\title{
Classification of Myocardial Infarction using Convolution Neural Network
}

\author{
K.Venu, P.Natesan, B.Krishnakumar, N.Sasipriya
}

\begin{abstract}
Myocardial infarction is one of the most dangerous cardiovascular diseases for most of the people in the world. It is generally confessed as a heart attack. The damage of the heart muscle causes the Myocardial Infraction (MI). When there is a block in heart veins, then the flow of oxygen to the heart muscle also gets blocked, which leads to damage of the heart muscle. The damage is irreversible, so it may lead to death. Quick and exact recognition of $M I$ is required to reduce the death rate. There are several diagnostic tools such as blood tests, ECG is available for the analysis of cardiovascular disease. Among all tools, ECG provides effective results in determining MI, but the manual interpretation of the ECG signal may take time for the doctor to identify the symptoms of MI. The manual interpretation may vary from person to person. Hence a computer-aided diagnostic tool is required to analyze ECG signals effectively for identifying MI. This paper aims to provide an algorithm for the detection of myocardial infarction that operates directly on ECG data. Nowadays Convolutional neural network is cable of analyzing an image effectively so, a deep learning model with the CNN algorithm is used in this paper to classify the images and to identify whether the image has MI or not. The proposed CNN model yields $87 \%$ accuracy for the Physikalisch-Technische Bundesanstalt database.
\end{abstract}

Keywords: Convolution Neural Network, CNN, Deep Learning, Myocardial Infraction, Classification

\section{INTRODUCTION}

Routine blood supply is required for the heart muscle cells to perform its functionalities effectively. Coronary arteries in the heart are responsible for providing oxygenrich blood to the heart muscle. The expansion of plaques causes blockage in the coronary artery, which will cut down the amount of blood supplied to the heart muscle. Then that section of the myocardium is disturbed due to that lack of blood flow. If this insufficient blood supply is not rectified in time, then it will lead to the death of the muscle in that section. This condition is called as Myocardial Infraction.[11] The blockage of the arteries, which affects the normal functionality of the heart, is called as heart attack. MI is the indirect symptom of heart attack, and hence it is called a silent attack. Records say that $72 \%$ of the people die due to silent heart attack. Early detection of MI is essential in today's world to provide appropriate treatment and to save people's life. MI will be positioned in the former, edgeways, backside, or inferior walls of the left ventricle. MI can be determined using Electrocardiography (ECG). It is the simplest and extensively used technology. The ECG records of the electrical functionality of the heart as signals.

Revised Manuscript Received on November 15, 2019

* Correspondence Author

K.Venu*, Department of CSE,Kongu Engineering College,Erode, India. Dr.P.Natesan, Department of CSE,Kongu Engineering College,Erode, India.

B.Krishnakumar, Department of CSE,Kongu Engineering College,Erode, India.

N.Sasipriya, Department of CSE,Kongu Engineering College,Erode, India.
The ECG reads the electrical impulses in the heart that activate the heart muscle and its blood-pumping action. Twelve electrodes are affixed to the skin on the chest, arms and legs sense those impulses from various vantage points segments the ECG waves. The ECG segments the heart wave like $\mathrm{P}$ wave, QRS wave, and $\mathrm{T}$ wave. When an abnormality happens in the activity of the heart, then it will be reflected in the ECG signal. ECG provides information about the presence of Myocardial Infarction (MI), when there is a change in ST-Segment Elevation, Abnormal Q Wave Elevation, and T-Wave inversion. The 12 different type of ECG Signals are used by the CNN Model for analysing whether the person is affected by MI or not.

The deviations in the ECG signals can be identified by the cardiologist. But there exist several challenges in the visual interpretation of the signals. The ECG signals have small amplitude duration, so analysis may be timeconsuming and inter - person variability occurs. These shortcomings can be beaten by utilizing a computer-aided diagnosis system for automatic detection of myocardial infarction on ECG. In deep learning, CNN based approach that works with end-to-end architecture is used for automatic detection of myocardial infarction using standard ECG data. It provides fast and effective analysis of MI.

\section{LITERATURE SURVEY}

MI Identification system using one lead and singlechannel is proposed in [1]. Here a method is developed to study low pass filter. The primary objective function which is focused here is to develop a filter using which the features are extracted. K- Nearest Neighbour technique is used to detect MI using the features extracted using filters. Analyzing the presence of MI in the ECG signal is done in [2] by using beats, which is obtained by breaking up of ECG signals. Wavelet Transform is applied to every beat from which sub band signals are obtained. Then from this sub band, entropy is calculated, and it is given as input to several classification techniques such as random forest, decision tree, neural network, and Support Vector Machine. Then the accuracy of each methodology is compared. As a result, this study concludes that SVM performs better when compared to all other classification techniques. Comparative analysis has been performed in [3] based on ECG acquisition, Feature extraction techniques, and ECG analysis techniques.

Various transforms such as contourlet, wavelet, and shearlet where used in [4] to extract the features , scolgrams, and entropies from the given ECG images. PSO is used to select only the features which are significant from the extracted features. Then the chosen features are ranked by utilizing its variance. Then the features where given as input to various classifiers such as decision tree and $\mathrm{k}$ nearest neighbour classifiers for classifying different types of abnormalities such as heart attack, MI and heart disease. 


\section{Classification of Myocardial Infarction using Convolution Neural Network}

In [5] new features are extracted from multi-lead ECG signals for identification of heart disease. The new feature is based on sample entropy upon using this, reduces the number of other features used for the analysis. It will contain all the information needed for the detection of heart disease. The achievement of this parameter is determined by using it in Support Vector Machine for the classification of various diseases. The proposed method provides high accuracy.

Usually, 2 dimensional ECG signals will be taken for analysis to determine whether that particular person is affected by MI or not. In [6] three dimensional information from the ECG signal is used. The higher-order dimensional will reveal the dependency between the beats, so that it will be able to produce more accurate results. In this work also support vector machine is used for classification and it produces good results. In the past study [7-9] both linear features and nonlinear features are generated from the ECG signal by utilizing various feature extraction techniques. A large number of features were generated as output,So, feature selection or dimensionality reduction techniques were used to reduce the amount of features used for analysis. Then the features are given as input to various classifiers for the purpose of classification.

In all these study features are generated using a methodology that takes time for extraction. So in this paper convolution neural network is used for classification. Here there is no need of pre-processing for feature extraction.

\section{A. ECG Signal}

\section{METHODOLOGY}

The ECG Signal consists of PQRST Waves to represent the activity of the heart. The $\mathrm{P}$ wave represents atrial contraction. It records the electrical activity of the heart muscles when the blood flows from atrial to ventricles. P- Wave will be $2.5 \mathrm{~mm}$ tall and it lasts for 0.11 seconds. The QRS waves consist of $\mathrm{Q}$ wave, $\mathrm{R}$ Wave, $\mathrm{S}$ wave. It represents the ventricular contraction that makes the blood flow from ventricles to the right atrium. The $\mathrm{T}$ wave represents ventricular relaxation. ST section in the PQRST wave represents the time duration between the ventricular depolarization and repolarization. MI affect person will have the symptom of ST-Segment Elevation, Abnormal Q Wave Elevation and T-Wave inversion in their ECG. In order to extract these features from the given input image and to classify the image, convolution neural network architecture is used.

\section{B. Convolution Neural Network}

The process involved classifying the image using CNN is shown in Fig.1. The dataset is divided into two parts training and testing dataset. Training dataset is used to train and build the model. The trained model is used by the test dataset to categorize it as either MI affected or healthy

Convolution Neural Network(CNN) is one of the most recently used deep learning techniques[12]. It has its wide range of application in image recognition, classification, object detection, etc., Images in the training set is given as input to CNN Model for training the model. In the training phase, the learnable parameters such as weights and bias will be updated. During the training process the features are automatically extracted by the CNN model. Filters play an important role in $\mathrm{CNN}$ for finding the spatial and temporal dependencies from the given image. It makes CNN more flexible when compared to other techniques. Once the training process gets completed, a test data without class label is given as input to the model and it will generate the class label as its output.

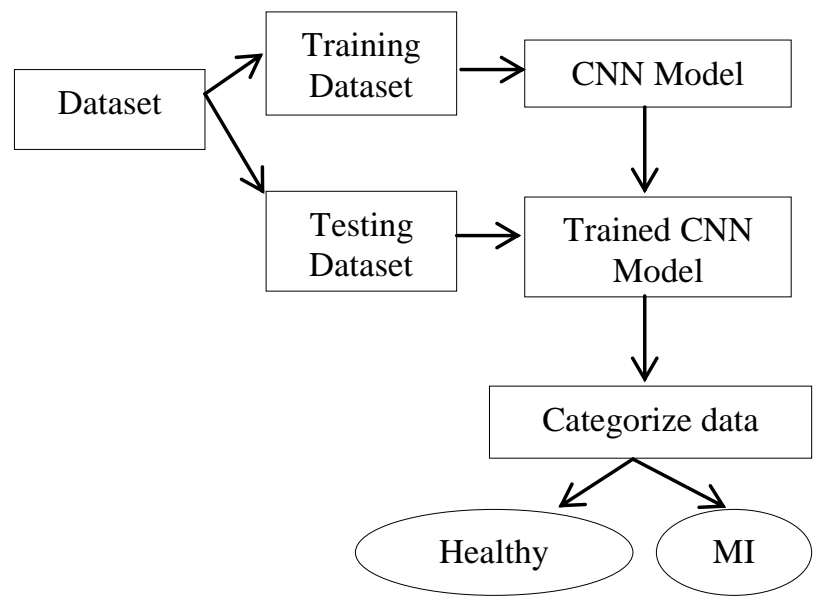

Fig.1. System Design

The input image given to CNN Model will be sent through the layers such as Convolution Layer followed by Pooling Layer and the last Fully Connected Layer. The purpose of each layer is given below.

\section{- Convolution Layer}

The first layer in the $\mathrm{CNN}$ model is the convolution layer. It is responsible for extracting both high level and low -level features such as edges, color, gradient orientation, etc. The images can also be made blur and sharpen in this layer. Here the features are learned from the given sample using input filters. Kernels is also called as weight/Filter. The number of kernels used in a convolution layer is equal to the number of features extracted. In the convolution layer the relationship between pixels is also conserved using a mathematical operation between the input image and the kernel. There can be N Number of convolution layers. The output of the convolution layer is convolved features.

\section{- Padding Layer and ReLU Activation Function}

The dimension of the convolved features is less when compared to the input image. So if the dimension is to be increased or make it equal to the input image padding is done. Non- Linear process is carried out by Rectified Linear Unit ( ReLu) in order to bring out non- linearity in convolution layer. When a set of numerical value is given as input to ReLU function it will provide only positive values as output. Negative values from the input will be discarded and it is made as zero by Relu. Since CNN has to learn only positive linear values for the real-world dataset. $\mathrm{ReLu}$ activation function is the most effective one when compared to other functions such as sigmoid and tanh. So ReLU activation function is used in all the convolution Layer of the CNN Model.

\section{- Pooling Layer}

Feature maps, which is the output from the convolution layer is given as input to the pooling layer. This layer performs its operation on each feature map one by one. Here the dimension of the input image is diminished in order to decrease the computing time and the number of parameters 
used. Even though the dimensions are reduced using pooling the information will the retained. There are various types of pooling such as Max Pooling which takes the maximum value, Sum Pooling where the sum of the values are calculated and similarly Average Pooling. A small matrix size will be predefined. That matrix space values will be taken at a time and if max pooling is used then maximum element in the take matrix of the input will be extracted. Then the matrix will be traversed to the next position and the operation continues. As a result for each matrix one element will be taken. In case of average pooling, average of all the element present in the matrix space will be given as output.

\section{- Fully Connected Layer}

Both convolution and pooling layer put together forms a single layer. Like this several layers will be created in order to develop a model and the last layer is fully connected layer, which is similar to the neural network. Features are extracted from the input image and the model has learnt the features using all the convolutional layers. The result of convolutional layer is given as input to fully connected layer for learning the non-linear sequence of the features.T he output image from convolution layer is flattened as column vector and it is given as input to the fully connected feed forward neural network. The final layer of the Multi-layer neural network is the output layer. The number of neurons is the output layer is equal to the number of output categories. In this layer softmax activation function is used to produce the output of each neuron. The category corresponding to the neuron with highest value is given as output.

\section{- Batch Normalization}

Input information are pre-processed while it is used for training the network model. Normal distribution is performed to normalize the input data. Normalization ensures that the input values of the first layer are in specified range. But the output of the input layer after activation is passed to the next hidden layer so no standard normalization is provided in hidden layers because the activation function varies from layer to layer. In the training process based upon the distribution, each layer has to separately prepare themselves for the purpose of learning. Hence internal covariate shift problem arises and the computation time gets increased. So in deep learning a technique to standardize the input values in the hidden layer is used, which is called as batch normalization. Here normalization is done by calculating batch mean and batch standard deviation. In each hidden layer ,the output of the previous layer is processed by using mean and standard deviation to find a normalized value. Mean and variance are the learnable parameters hence its values get trained during the training process. Then during testing is made fixed. As a result, stability of the model gets improved, fast convergence rate is achieved with less computation time. Moreover batch normalization helps the network to learn independently without depending upon other layers.

\section{- Dropout}

When smaller dataset is used for training convolution neural network model then overfitting of the data which is used for training will happen. Overfitting will occurs, when the model tries to memorize the data or when it tries the to learn the noise present in the training data. Overfitting

increases the training accuracy whereas the testing accuracy will be decreased. It will reduce the performance of the model when it works for new dataset. As a result generalization error gets increased. In order to decrease overfitting, dropout which is a regularization technique is used. In dropout, random number of neurons in each hidden layer will be left out for calculations during the forward and backward pass of the training process. During each different passes distinct neurons will be left out. When a neuron is dropped then its incoming and outgoing edge is also detached. Each neuron is dropped with some probability value. So network architecture will vary in each training pass which will reduce the overfitting considerably. Dropout also helps the network model to learn the sparse representation.

\section{- Adam Optimizer}

The main objective of the machine learning algorithm is to reduce the value of loss function. The loss function calculates the difference between predicted output of the given input with the actual output of that particular sample. Optimal weight values is to be found in order to decrease the value of the cost function and to increase the generalization which in turn increases the performance of the model. The weight values will get updated using the optimizers based on the output of the loss function. Adam is the most widely used optimization algorithm for training the convolution neural network. Adam is designed by combining the benefits of both RMSprop optimizer and stochastic Gradient descent optimizer which is used along with momentum. Learning rate is the parameter using which the weight values gets updated iteratively based on the training data. In some of the optimizers this learning rate value is fixed and it doesn't change over iterations. In Adam technique it is learnable and it varies adaptively for each individual parameters of the model. Learning rate is calculated using the value of the first moment and average of the second moment. Adam will obtain optimal value quickly hence the convergence time will be very less compared to other optimizers.

\section{PROPOSED CNN MODEL}

\section{A. Training Phase}

Training process of the convolution neural network model consist of the following two passes, Forward pass followed by Backward pass. During the forward pass the input image is passed through all the layers of CNN model and final output will be calculated. In backward pass based upon the cost function value the weights will be updated by the optimizers. Then the training continues for the next image in training dataset. Based upon the number of epochs the training of the model with the whole training dataset is done to make the model to learn the images by extracting the features.

\section{B. Testing Phase}

In testing phase a new image will be given as input to the Convolution Neural Network Model which is already trained. Using the learnable parameters such as weigh, learning rate, etc., the given image will be classified by the model either as healthy or MI affected.

\section{Model}




\section{Classification of Myocardial Infarction using Convolution Neural Network}

In the proposed model total seven convolution layers along with pooling layer is used. The input image is taken with 128 X 128 Pixels. Filter size of each layer varies in order to incorporate the up sampling and down sampling for feature extractions. Adam optimizer is used in this proposed work for updating of learnable parameters. Since the number of output class is two binary cross-entropy is used as loss function to calculate the difference between the actual and predicted output

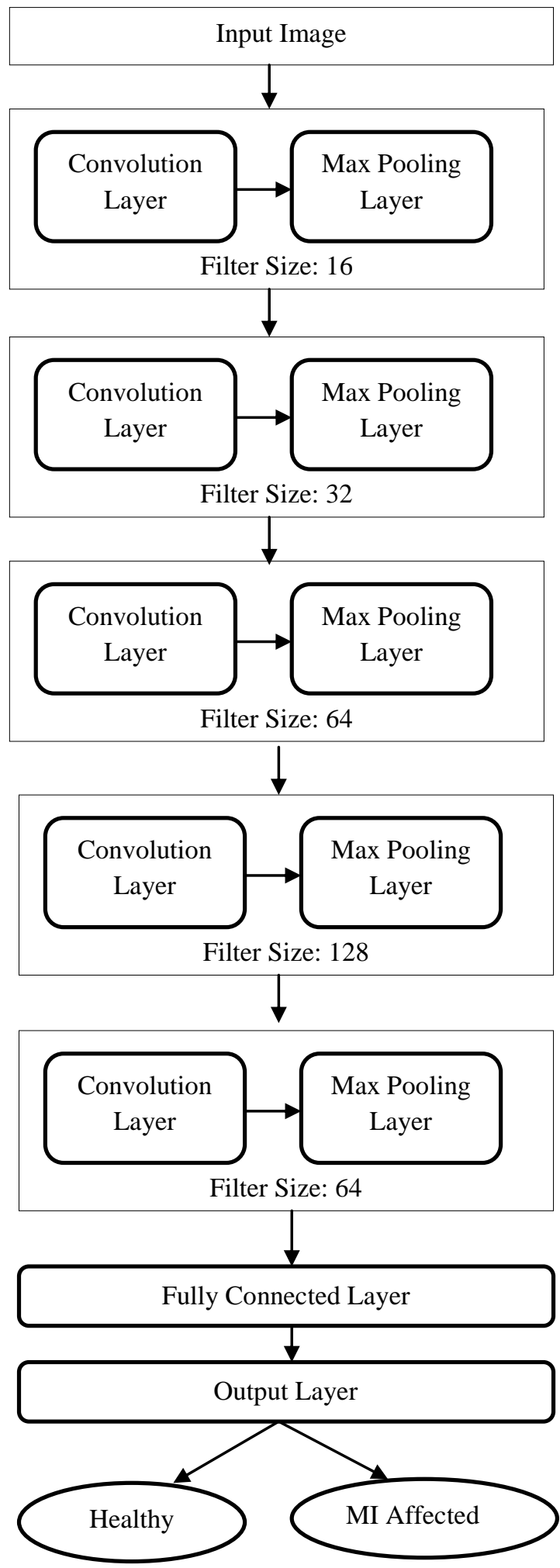

Fig. 2. Proposed System Architecture
Finally a fully connected layer with 512 neurons are used to combine all the features extracted by the convolution layers.Then the output layer contains two neurons as the dataset is going to classified as two categories such as Healthy and MI affected. The proposed CNN Model for classifying the given image is shown in Fig.2

The input to the CNN Model will the 12 Lead ECG Signal of a particular person. During the training process the features such as P-Peak, R- Peak, interval and durations will be automatically extracted and learnt by the CNN Model without any preprocessing work. Using theb initial layers low level features are extracted and then it extracts the high level features that helps in the process of classification during the testing phase.

\section{DATASET DESCRIPTION}

The Dataset is collected from the ECG Signal Repository [10]. Actual Data set downloaded from the repository consist if 182 Healthy ECG Signal and 366 MI Affected person's ECG Signal. In order to make the model more accurate. Augmentation process is carried out and the 450 Healthy ECG Signal and 720 MI affected signals are obtained and it is used for training and testing process.

\section{A. Experimental Setup}

The number of training and testing images used for training and testing of proposed CNN Model is shown in Table - I.

Table - I:No. of Training and Test Images

\begin{tabular}{|l|l|l|}
\hline Category & $\begin{array}{l}\text { No. of Training } \\
\text { Images }\end{array}$ & $\begin{array}{l}\text { No. of Testing } \\
\text { Images }\end{array}$ \\
\hline Healthy & 400 & 50 \\
\hline MI Affected & 470 & 50 \\
\hline
\end{tabular}

Training images from both the categories are used to train the model. Totally 15 epochs are used to train the model. Testing images are used to test the model. The proposed CNN Model shows better results.

\section{RESULTS AND DISCUSSION}

Number of True Negative sample are the number of samples which are actually negative and predicted category by CNN is also Negative. For the given data set Number of True Negative are the number samples which are actually healthy and predicted category is also Healthy. Number of True positive sample are the number of samples which are actually positive and predicted category by $\mathrm{CNN}$ is also positive. For the given data set Number of True Positive are the number samples which are actually MI affected and predicted category is also MI Affected.

Number of False Positive sample are the number of samples which are actually negative and predicted category by CNN is positive. For the given data set Number of False Positive are the number samples which are actually healthy and predicted category is MI Affected. Number of False Negative sample are the number of samples which are actually positive and predicted category by $\mathrm{CNN}$ is Negative. For the given data set Number of False Positive are the number samples which are actually MI affected and predicted category is healthy. The confusion matrix for the give test ECG signal data is shown in the Table-II. 
Table-II. Confusion Matrix

\begin{tabular}{|l|l|c|c|}
\cline { 3 - 3 } \multicolumn{2}{c|}{} & \multicolumn{2}{|c|}{ Predicted } \\
\cline { 3 - 4 } \multicolumn{2}{c|}{ Actual } & Healthy & MI Affected \\
\cline { 2 - 4 } & Mealthy & 38 & 12 \\
\cline { 2 - 4 } & MI Affected & 6 & 44 \\
\hline
\end{tabular}

\section{A. Precision}

The precision score for MI Affected gives the ratio between the number of sample correctly classified as MI Affected and Total number of samples classified as MI Affected by the model.The precision score for Healthy gives the ratio between the number of sample correctly classified as Healthy and Total number of samples classified as Healthy by the model.

\section{B. Recall}

The Recall score for MI Affected gives the ratio between the number of sample correctly classified as MI Affected and Total number of samples which are actually MI Affected.The Recall score for Healthy gives the ratio between the number of sample correctly classified as Healthy and Total number of samples which are actually Healthy.The Precision and recall for two different categories are shown in the following Table- III.

Table-III. Precision and Recall Values

\begin{tabular}{|l|c|c|}
\hline Category & Precision & Recall \\
\hline Healthy & 0.86 & 0.76 \\
\hline MI Affected & 0.78 & 0.88 \\
\hline
\end{tabular}

The training accuracy during 15 Epochs are shown in the following Fig. 3

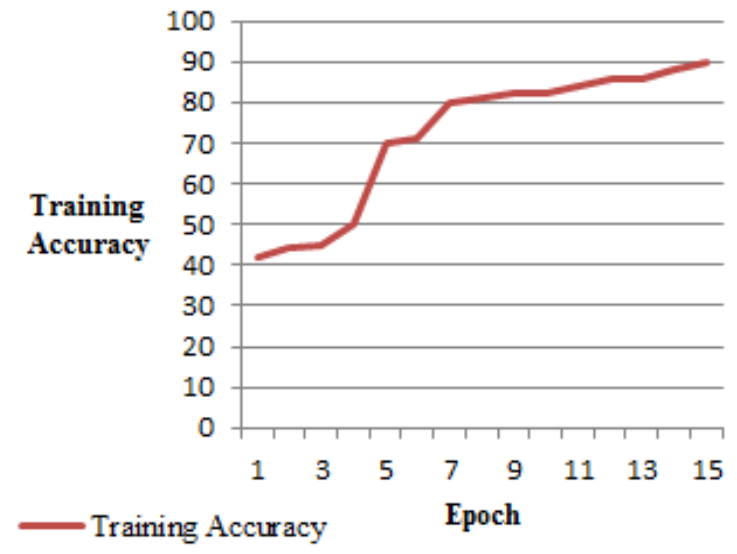

Fig.3. Training Accuracy Vs Epoch

The testing accuracy of the proposed model is found to be $82 \%$. Eventhough the accuracy is less when compared to the previous study. In this proposed system the features are automatically extracted so the computation time is considerably reduced.

\section{CONCLUSION}

In this proposed system Convolution Neural Network which is a deep Learning technique is used for classification. The training and testing process of the proposed 5 layer $\mathrm{CNN}$ model along with max pooling layer and fully connected layer is done by using ECG images for classifying it as MI affected or healthy. This model yielded $82 \%$ accuracy. In this proposed method, no pre-processing work for feature extraction is needed as it is done automatically by CNN. This model can be applied for automated MI detection on
ECG images. In future different hyper-parameters of CNN model such as filter value, number of hidden layers will be optimized in order to obtain good accuracy.

\section{REFERENCES}

1. M. Sharma, "A novel automated diagnostic systemfor classification of myocardial infarction ECG signals using an optimal biorthogonal filter bank", Computers in Biology and Medicine,2018,pp. 341-356

2. M.Kumar," Automated diagnosis of myocardial infarction ECG signals using sample entropy in flexible analytic wavelet transform framework. Entropy", Computers in Biology and Medicine,2017,pp. 488-492.

3. J.T.Weng, "Myocardial infarction classification by morphological feature extraction from big 12-lead ECG data.,,In Pacific- Asia Conference on Knowledge Discovery and Data Mining, Springer, 2014.pp. 689-699.

4. U.R.Acharya," Automated characterization of coronary artery disease, myocardial infarction, and congestive heart failure using contourlet and shearlet transforms of electrocardiogram signal", Knowledge-Based Systems, 132,2017, pp. 156-166.

5. R.K.Tripathy," A new way of quantifying diagnostic information from multilead electrocardiogram for cardiac disease classification",Healthcare Technology Letters, 2014, pp. 98-103.

6. S.Padhy, " Third-order tensor based analysis of multilead ECG for classification of myocardial infarction",Biomedical Signal Processing and Control, 2017,pp. 71-78.

7. H.Fujita, "Characterization of cardiovascular diseases using wavelet packet decomposition and nonlinear measures of electrocardiogram signal", In International Conference on Industrial, Engineering and Other Applications of Applied Intelligent Systems, Springer, 2017. pp. 259-266.

8. T.Kenttä, "Prediction of sudden cardiac death with automated highthroughput analysis of heterogeneity in standard resting 12-lead electrocardiograms",Heart Rhythm, 13(3),2016,pp. 713-720.

9. R.Tao," Magnetocardiography based ischemic heart disease detection and localization using machine learning methods", IEEE Transactions on Biomedical Engineering, 2018.

10. https://archive.physionet.org/cgi bin/atm/ATM

11. U.R. Acharya, N. Kannathal, M.H. Lee, M.Y. Leong, "Study of heart rate variability signals at sitting and lying postures" J. Body Mov. Ther., 2005, pp. 134-141

12. I. Goodfellow, Y. Bengio, A. Courville Deep Learning MIT Press 2016.

\section{AUTHORS PROFILE}

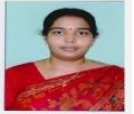

Ms. K.Venu is currently working as a Assistant Professor in the department of Computer Science \& Engineering in Kongu Engineering College, Tamilnadu, India. She is pursuing Ph.D., in Machine Learning under Anna University. She has completed 2 years of teaching service. She has published 3 articles in International/ National Conference. She has authored 1 book chapter with reputed publishers

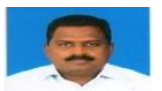

Dr.P.Natesan has completed his Ph.D., degree in Network security in 2013 in Anna University. Currently he is a Professor in the department of Computer Science \& Engineering in Kongu Engineering College, Tamilnadu, India. He has completed 22 years of teaching service. He has published 17 articles in International /National Journals. He has authored 2 book chapter with reputed publishers. $\mathrm{He}$ is serving as a Editorial/Advisory board member for many Journals. He has completed 4 funded projects and organized 7 funded seminars and workshops.

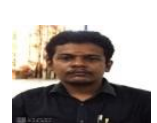

Mr. B.Krishnakumar is pursuing Ph.D., in Machine Learning under Anna University. Currently he is a Assistant Professor in the department of Computer Science \& Engineering in Kongu Engineering College, Tamilnadu, India. He has completed 11 years of teaching service. He has published 5 articles in International \&National Journals. He has published 9 articles in International \& National Conference. He has organized 2 funded seminars and workshops. He has authored 1 book chapter with reputed publishers 


\section{Classification of Myocardial Infarction using Convolution Neural Network}

Ms.N.Sasipriyaa is currently working as a Assistant Professor in the department of Computer Science \& Engineering in Kongu Engineering College, Tamilnadu, India. She has completed 11 years of teaching service. She has published 4 articles in International \& National Journals. She has published 8 articles in International \& National Conference. She has organized 1 funded workshop. She has authored 1 book chapter with reputed publishers. 